DAMTP-2002-137

\title{
Polyhedral Scattering of Fundamental Monopoles
}

\author{
Richard A. Battye ${ }^{1}$, Gary W. Gibbons ${ }^{2}$, \\ Paulina Rychenkova ${ }^{3}$ and Paul M. Sutcliffe ${ }^{4}$ \\ 1 Jodrell Bank Observatory, Macclesfield, Cheshire SK11 9DL U.K. \\ \& Department of Physics and Astronomy, Schuster Laboratory, \\ University of Manchester, Brunswick St, Manchester M13 9PL, U.K. \\ Email : rbattye@jb.man.ac.uk \\ 2 Department of Applied Mathematics and Theoretical Physics, \\ Centre for Mathematical Sciences, University of Cambridge, \\ Wilberforce Road, Cambridge CB3 0WA, U.K. \\ Email : G.W.Gibbons@damtp.cam.ac.uk \\ ${ }^{3}$ Charles River Ventures, 1000 Winter St, Waltham, MA 02451 USA. \\ Email : paulina@crv.com \\ ${ }^{4}$ Institute of Mathematics, University of Kent at Canterbury, \\ Canterbury, CT2 7NZ, U.K. \\ Email : P.M.Sutcliffe@ukc.ac.uk
}

November 2002

\begin{abstract}
The dynamics of $n$ slowly moving fundamental monopoles in the $S U(n+1)$ BPS Yang-Mills-Higgs theory can be approximated by geodesic motion on the $4 n$ dimensional hyperkähler Lee-Weinberg-Yi manifold. In this paper we apply a variational method to construct some scaling geodesics on this manifold. These geodesics describe the scattering of $n$ monopoles which lie on the vertices of a bouncing polyhedron; the polyhedron contracts from infinity to a point, representing the spherically symmetric $n$-monopole, and then expands back out to infinity. For different monopole masses the solutions generalize to form bouncing nested polyhedra. The relevance of these results to the dynamics of well separated $S U(2)$ monopoles is also discussed.
\end{abstract}




\section{Introduction}

The dynamics of slowly moving BPS monopoles can be approximated by geodesic motion on the moduli space of static solutions, with the metric determined by the kinetic part of the Lagrangian [21, 24]. For two centred $S U(2)$ monopoles the moduli space is the Atiyah-Hitchin manifold and the simplest geodesic corresponds to the $90^{\circ}$ scattering of two monopoles in a head-on collision [3]. Unfortunately for more than two $S U(2)$ monopoles the moduli space metric is not known explicitly, except in the region where all the monopoles are well separated [13]. Despite this fact some geodesics are known [14, 16, 17, 25]. They are obtained by the imposition of appropriate spatial symmetries to yield a one-dimensional manifold of static solutions, which is then automaticaly a geodesic, since the fixed point set of a group action is always a totally geodesic submanifold.

For BPS monopoles with gauge group $S U(n+1)$ and maximal symmetry breaking there are $n$ topological charges and correspondingly $n$ types of fundamental monopole, each of which carries a single unit of one of these charges [26]. If there is precisely one fundamental monopole of each type then the moduli space is $4 n$-dimensional and equiped with the hyperkähler Lee-Weinberg-Yi metric [19], which is known explicitly. The explicit form of the metric allows the possibility of computing some geodesics and hence $n$-monopole scattering processes for any value of $n$. In this paper we apply a variational method to construct some scaling geodesics on this manifold. The approach is to look for central configurations in which the time dependence is only in the form of an overall scaling of the monopole positions. The resulting algebraic equations can then be written as the critical points of a certain energy function [23], which we minimize using numerical methods. As examples, we find minimal energy configurations, and hence geodesics, for all $n \leq 20$. The symmetries of these configurations are analyzed and suggest the existence of icosahedral minima at $n=32$ and $n=72$, which are also constructed. In all cases the associated geodesics describe the scattering of $n$ monopoles which lie on the vertices of a bouncing polyhedron, in the following sense. The polyhedron first contracts from infinity to a point, which in the moduli space represents the spherically symmetric $n$-monopole. The evolution then reverses with the monopoles located on the vertices of the same polyhedron, but which is now expanding back out to infinity.

For different monopole masses the above solutions generalize to form bouncing nested polyhedra. Our solutions also provide geodesics of the Gibbons-Manton metric [13], and hence describe the scattering of $S U(2) n$-monopoles, valid in the region where the monopoles are well-separated. This reveals a connection with some geodesics obtained earlier using symmetry arguments.

\section{Polyhedral Scattering}

The $4 n$-dimensional hyperkähler Lee-Weinberg-Yi manifold is a $T^{n}$ bundle over a $3 n$ dimensional base space. For $i=1, . ., n$ let $\theta_{i} \in S^{1}$ be the fibre coordinates and $\mathbf{x}_{i} \in \mathbb{R}^{3}$ be local coordinates in the base, which may be thought of as the positions in $\mathbb{R}^{3}$ of each of 
the $n$ monopoles. The purely kinetic Lagrangian associated with the metric has the form

$$
L=g_{i j} \dot{\mathbf{x}}_{i} \cdot \dot{\mathbf{x}}_{j}+g_{i j}^{-1}\left(\dot{\theta}_{i}+\mathbf{W}_{i k} \cdot \dot{\mathbf{x}}_{k}\right)\left(\dot{\theta}_{j}+\mathbf{W}_{j l} \cdot \dot{\mathbf{x}}_{l}\right),
$$

where we have used the Einstein summation convention, though this is not to be used in the rest of the paper unless explicitly stated. The quantities appearing in the above are given by

$$
\begin{aligned}
g_{i i} & =m_{i}+\sum_{j \neq i} \frac{1}{\left|\mathbf{x}_{i}-\mathbf{x}_{j}\right|}, \\
g_{i j} & =\frac{-1}{\left|\mathbf{x}_{i}-\mathbf{x}_{j}\right|}, \quad i \neq j \\
\mathbf{W}_{i i} & =\sum_{j \neq i} \mathbf{w}_{i j}, \\
\mathbf{W}_{i j} & =-\mathbf{w}_{i j}, \quad i \neq j
\end{aligned}
$$

and $\mathbf{w}_{i j}$ is the value at $\mathbf{x}_{i}$ of the Dirac potential due to the monopole at $\mathbf{x}_{j}$, that is

$$
\nabla_{j} \times \mathbf{w}_{j i}=\frac{\mathbf{x}_{i}-\mathbf{x}_{j}}{\left|\mathbf{x}_{i}-\mathbf{x}_{j}\right|^{3}}
$$

In the above we have scaled out the magnetic charge of a monopole and the positive constants $m_{i}$ are related to the monopole masses.

The $T^{n}$ isometry of the metric yields the $n$ conserved charges (here we use the summation convention once more)

$$
Q_{i}=g_{i j}^{-1}\left(\dot{\theta}_{j}+\mathbf{W}_{j k} \cdot \dot{\mathbf{x}}_{k}\right)
$$

so that the fibre coordinates are non-dynamical degrees of freedom. In this paper we shall be concerned with monopoles with no electric charge, so we set $Q_{i}=0$, for all $i=1, . ., n$. In this case the Lagrangian describing the motion in the base space is simply

$$
L=\sum_{i}\left(m_{i}+\sum_{j \neq i} \frac{1}{x_{i j}}\right) \dot{\mathbf{x}}_{i}^{2}-\sum_{i} \sum_{j \neq i} \frac{1}{x_{i j}} \dot{\mathbf{x}}_{i} \cdot \dot{\mathbf{x}}_{j}
$$

where we have defined $\mathbf{x}_{i j}=\mathbf{x}_{i}-\mathbf{x}_{j}$, and $x_{i j}=\left|\mathbf{x}_{i j}\right|$. The geodesic equations which follow from (2.8) are

$$
m_{k} \ddot{\mathbf{x}}_{k}=\sum_{j \neq k}\left(\frac{\ddot{\mathbf{x}}_{j k}}{x_{j k}}+\frac{\mathbf{x}_{j k}\left|\dot{\mathbf{x}}_{j k}\right|^{2}}{2 x_{j k}^{3}}-\frac{\dot{\mathbf{x}}_{j k} \dot{x}_{j k}}{x_{j k}^{2}}\right),
$$

As suggested in [23] we now look for time-dependent homothetic solutions of these equations, that is, solutions of the form $\mathbf{x}_{k}(t)=\alpha(t) \mathbf{y}_{k}$, with constant $\mathbf{y}_{k}$. Clearly, such solutions describe monopoles in a fixed configuration, but with the overall scale of the configuration evolving dynamically. Substituting this ansatz into (2.9) yields the equations

$$
m_{k} \mathbf{y}_{k}+C \sum_{j \neq k} \frac{\mathbf{y}_{j k}}{y_{j k}}=0
$$


where $C$ is defined to be the quantity

$$
C=\frac{\dot{\alpha}^{2}}{2 \ddot{\alpha} \alpha^{2}}-\frac{1}{\alpha} .
$$

Obviously, for a non-trivial solution of (2.10) to exist the quantity $C$ must be a constant, and it turns out that only a positive constant produces a physically acceptable solution. By a rescaling of the time variable we may, without loss of generality, set $C=1$. The two constants which appear in the general solution of (2.11) may be absorbed by a linear transformation of $t$, and the solution we require is given implicitly by

$$
t=\sqrt{\alpha+\alpha^{2}}+\frac{1}{2} \log \left(1+2 \alpha+2 \sqrt{\alpha+\alpha^{2}}\right)
$$

for $t \geq 0$. It is clear from (2.12), that the scale $\alpha(t)$ is a monotonically increasing function of $t$, which for small $t$ has the expansion $\alpha=t^{2} / 4+\ldots$ and for large $t$ has the asymptotic form $\alpha \sim t$.

Note that equations (2.9) are invariant under time-reversal $t \mapsto-t$ and also spatial inversion of all the points $\mathbf{x}_{k} \mapsto-\mathbf{x}_{k}$. So far we have only addressed the second part of the geodesic motion, when $t \geq 0$ and the monopoles are moving away from each other, but the first part with $t<0$ is simply obtained by time-reversal in equation (2.12), so that the monopoles approach the origin from spatial infinity. Thus the monopoles bounce back off each other, rather than passing through each other, which would have been the result if the time inversion was accompanied by the spatial inversion $\mathbf{x}_{k} \mapsto-\mathbf{x}_{k}$. The fact that the first of these scenarios is the correct one can be seen by studying the Lee-Weinberg-Yi manifold in the neighbourhood of the origin $\mathbf{x}_{k}=0$, for all $k$. Although the metric appears to be singular at the origin, this is merely a coordinate singularity, and if new coordinates are chosen appropriately (these are essentially polar coordinates but with the radial variables related to the monopole positions by $r_{k}=\sqrt{\left|\mathbf{x}_{k}\right|}$ ) the metric is seen to be flat in these new coordinates. The fact that the squares of the monopole positions are related to the flat coordinates is the reason that fundamental monopoles of different types bounce back upon collision; had the metric been flat around the origin in the coordinates $\mathbf{x}_{k}$ then the monopoles would have passed through each other.

The problem of finding scaling geodesics has now been reduced to the algebraic problem of finding sets of $n$ points $\mathbf{y}_{k}$, which satisfy (2.10) with $C=1$. Our method is to use a variational approach, based on the fact that (2.10) are the equations for critical points of the energy function

$$
E=\frac{1}{2} \sum_{i} m_{i}\left|\mathbf{y}_{i}\right|^{2}-\sum_{i} \sum_{j<i} y_{i j} .
$$

In this formulation the problem has obvious similarities with the classical problem of finding central configurations [5] (which arise in a similar way when a time-dependent homothetic ansatz is used in Newton's equations of motion for gravitating point particles) or equivalently solutions of the One Component Plasma (OCP) model [6]. The OCP model describes point charges immersed in a uniform background of charge with the opposite 
sign. Hence there are two competing forces, the first is an attraction towards the origin, represented by exactly the same expression as the first term in (2.13), and repulsion between the points, which in the OCP case is described by the Coulomb energy. The second term in (2.13) plays a similar role in our problem as the Coulomb energy does in the OCP model. The contribution of this non-positive term produces two-body particle repulsions which can balance the attractive central force, producing stable minimal energy configurations with finite non-zero separations.

In the remainder of this section we shall restrict to the case when all the monopole masses are equal. By rescaling the positions $\mathbf{y}_{k}$ by the inverse of this common mass we obtain the situation in which all monopole masses are equal to unity, so for the rest of this section we set $m_{i}=1$ for all $i=1, \ldots, n$.

Although any critical point of the energy (2.13) will provide us with a geodesic on the Lee-Weinberg-Yi manifold we shall concentrate only on local minima, since these are the easiest to find numerically, and ignore any saddle point solutions. Presumably Leech's symmetric configurations [20], which are sets of particles on a sphere in equilibrium under any force law between pairs of particles, will also yield critical points of (2.13) if the particles are allowed to move off the sphere, but are required to maintain all symmetries of the spherical configuration. Leech's configurations consist of an infinite family of polygons and bipyramids and a finite family with Platonic symmetry.

The numerical scheme employed is a multi-start gradient flow algorithm with randomly distributed initial conditions. The energy function (2.13) has the obvious $S O(3)$ invariance associated with a spatial rotation of all $n$ points, and also reflection symmetries which change the sign of any one of the three Cartesian components of all the points. Up to the action of these symmetry groups, all the minimal energy solutions we find are unique.

The case $n=1$ is trivial; the minimal energy solution is a single point at the origin, with $E=0$, and hence the scaling solution is time-independent, so no geodesic is obtained. For two points the minimal energy is $E=-1$ which occurs if $\mathbf{y}_{1}=-\mathbf{y}_{2}=(0,0,1)$, or any spatial rotation of this configuration. In other words, the two monopoles are at antipodal points on the unit sphere. The associated geodesic describes the head-on collision of two monopoles, in which the spherically symmetric 2-monopole is formed, after which the monopoles bounce back off each other. This scattering process was first described by Connell [9], who discovered that the metric on the centred moduli space of two different fundamental $S U(3)$ monopoles is Taub-NUT with a positive mass parameter.

In Table 1 we present, for $2 \leq n \leq 20$, the energy $E$ of the minimizing configuration, its symmetry group $G$, the distance from the origin of the closest point $r_{\min }$, and the distance from the origin of the furthest point $r_{\max }$.

In the examples in Table 1 where $r_{\min }$ is equal to $r_{\max }$ to the accuracy presented they are in fact precisely equal, indicating that all the points lie on the surface of a sphere of radius $r_{\min }=r_{\max }$. For all the other cases it can be seen that $r_{\min }$ and $r_{\max }$ are very close in value, showing that all $n$ points lie close to, but not exactly on, a sphere. As we shall see later, this feature appears to persist for arbitrarily large values of $n$, which contrasts sharply with traditional central configurations with a Coulomb interaction, where this property exists only for $n<13$ and beyond this value there are multiple shells [5]. It is interesting to note 


\begin{tabular}{|r|r|c|r|r|}
\hline$n$ & \multicolumn{1}{|c|}{$E$} & $G$ & $r_{\min }$ & $r_{\max }$ \\
\hline 2 & -1.0000 & $D_{\infty h}$ & 1.0000 & 1.0000 \\
3 & -4.5000 & $D_{3 h}$ & 1.7321 & 1.7321 \\
4 & -12.0000 & $T_{d}$ & 2.4495 & 2.4495 \\
5 & -24.5916 & $D_{3 h}$ & 3.1018 & 3.1592 \\
6 & -43.9706 & $O_{h}$ & 3.8284 & 3.8284 \\
7 & -71.0162 & $C_{1}$ & 4.4782 & 4.5635 \\
8 & -107.5011 & $D_{4 d}$ & 5.1841 & 5.1841 \\
9 & -154.5499 & $D_{3 h}$ & 5.8376 & 5.8718 \\
10 & -213.5297 & $D_{4 d}$ & 6.5099 & 6.5412 \\
11 & -285.6593 & $C_{2 v}$ & 7.1648 & 7.2435 \\
12 & -372.7470 & $Y_{h}$ & 7.8819 & 7.8819 \\
13 & -475.3419 & $C_{2 v}$ & 8.5186 & 8.5980 \\
14 & -595.4323 & $D_{6 d}$ & 9.2142 & 9.2749 \\
15 & -734.0923 & $D_{3}$ & 9.8771 & 9.9279 \\
16 & -892.7338 & $T$ & 10.5541 & 10.5925 \\
17 & -1072.6591 & $D_{5 h}$ & 11.2308 & 11.2368 \\
18 & -1275.2163 & $D_{4 d}$ & 11.8834 & 11.9107 \\
19 & -1501.5794 & $C_{2 v}$ & 12.5491 & 12.5987 \\
20 & -1753.4547 & $D_{3 h}$ & 13.2348 & 13.2518 \\
\hline
\end{tabular}

Table 1: For $2 \leq n \leq 20$ we list the energy $E$ of the minimizing configuration, its symmetry group $G$, the distance from the origin of the closest point $r_{\min }$, and the distance from the origin of the furthest point $r_{\max }$. 
that a scale invariant geometric energy function exists which also yields minimal energy configurations on a single shell for all numbers of points [4]. It would be interesting to try and classify the properties of interaction potentials which produce only a single shell.

In fig. 1 we present, for $3 \leq n \leq 20$, the minimal energy configurations of $n$ points by plotting spheres around each of the points and in fig. 2 we plot their energy as a function of $n$, for $n \leq 20$. In each case the diameter of the spheres is equal to the minimal separation between points, to emphasize the sphere packing behaviour. As seen from Table 1, the size of the configuration (as measured by $r_{\max }$ ) grows with $n$, so for clarity we do not display the configurations to scale. Three points lie on the vertices of an equilateral triangle, with edge length equal to 3 . For $n>3$ the points may be considered as forming the vertices of a polyhedron, which generically is a deltahedron, that is, all faces are triangular. For example, four points lie on the vertices of a tetrahedron with edge length equal to 4 . For $n=4,6,8,12$ all points lie exactly on the surface of a sphere, and in fact on the vertices of a tetrahedron, octahedron, square antiprism and icosahedron, respectively.

As can be seen from Table 1 and fig. 1 the points are often arranged symmetrically, though the case $n=7$ is rather anomalous. There is an obvious $D_{5 h}$ symmetric candidate for the minimal energy $n=7$ configuration, in which 5 points lie on the vertices of a regular pentagon and the two remaining points lie on the 5 -fold symmetry axis equidistant from the origin. This regular bipyramid is the obvious generalization of the minimal $n=5$ configuration, which is a bipyramid with a triangular base. However, the minimal energy solution for $n=7$ is a symmetry breaking perturbation of the bipyramid. There are points at the north and south poles of a sphere of radius 4.5635, and the remaining five points lie in a roughly pentagonal distribution, but all with slightly different heights above or below the equatorial plane and different distances from the origin, which range from 4.4782 to 4.4825 . Clearly this prohibits any exact symmetry, even reflection symmetries, so we label the symmetry group as $C_{1}$, indicating no point symmetries. As a check it is possible to minimize within the family of $D_{5 h}$ symmetric configurations, yielding an energy of $E=-71.0156$, which is indeed slightly higher than the asymmetric minimum with $E=-71.0162$.

To gain insight into minimizing the energy function (2.13) (with $m_{k}=1$ ) it is useful to consider the restricted problem in which all the points are constrained to lie on a sphere of a given radius $\rho$. The energy of this restricted problem is given by

$$
E_{\rho}=\frac{1}{2} \rho^{2} n+\rho U
$$

where

$$
U=-\sum_{i} \sum_{j<i}\left|\mathbf{Y}_{\mathbf{i}}-\mathbf{Y}_{\mathbf{j}}\right|,
$$

for $n$ points $\mathbf{Y}_{\mathbf{k}}=\mathbf{y}_{k} / \rho$ restricted to lie on the surface of the unit sphere. Minimization of the function $U$ for points on the unit sphere is a problem in discrete geometry which was first posed almost fifty years ago by Fejes Tóth [12]; though it is usually phrased in terms of maximizing the sum of the mutual separations $-U$. There are a number of theorems 

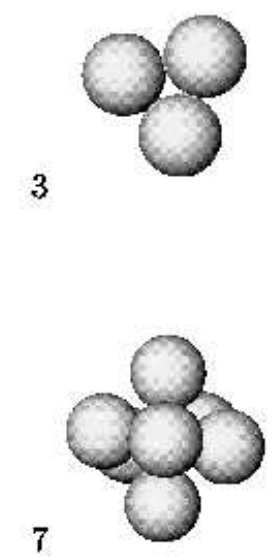

11

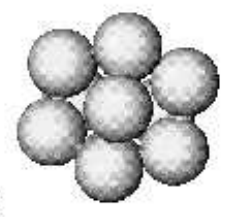

15

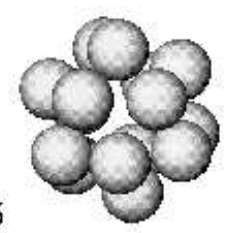

19

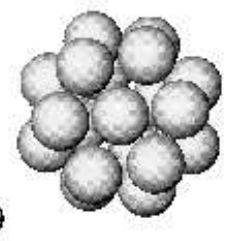

4

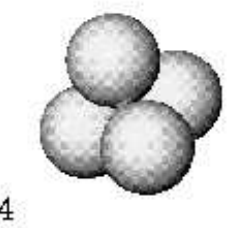

8

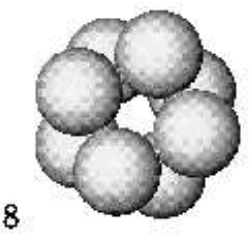

12

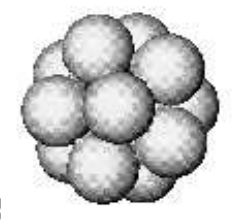

16
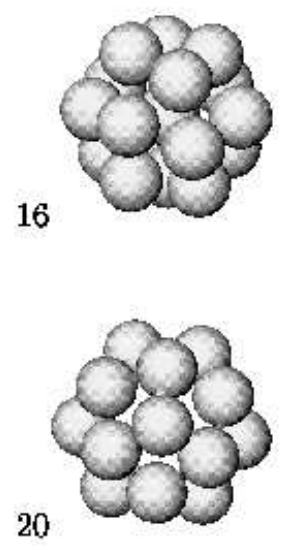

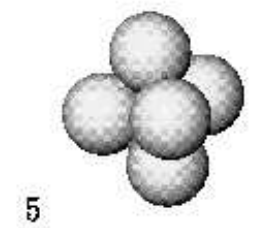

6

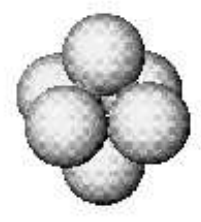

10

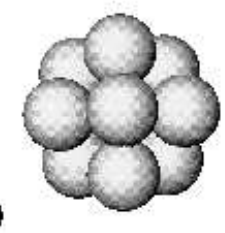

14

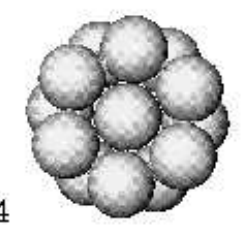

18

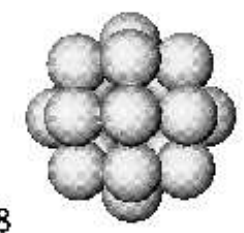

Figure 1: For $3 \leq n \leq 20$ we display the configurations of $n$ points (not to scale) by plotting spheres around each of the points. In each case the diameter of the spheres is equal to the minimal separation between points, to emphasize the sphere packing behaviour. 


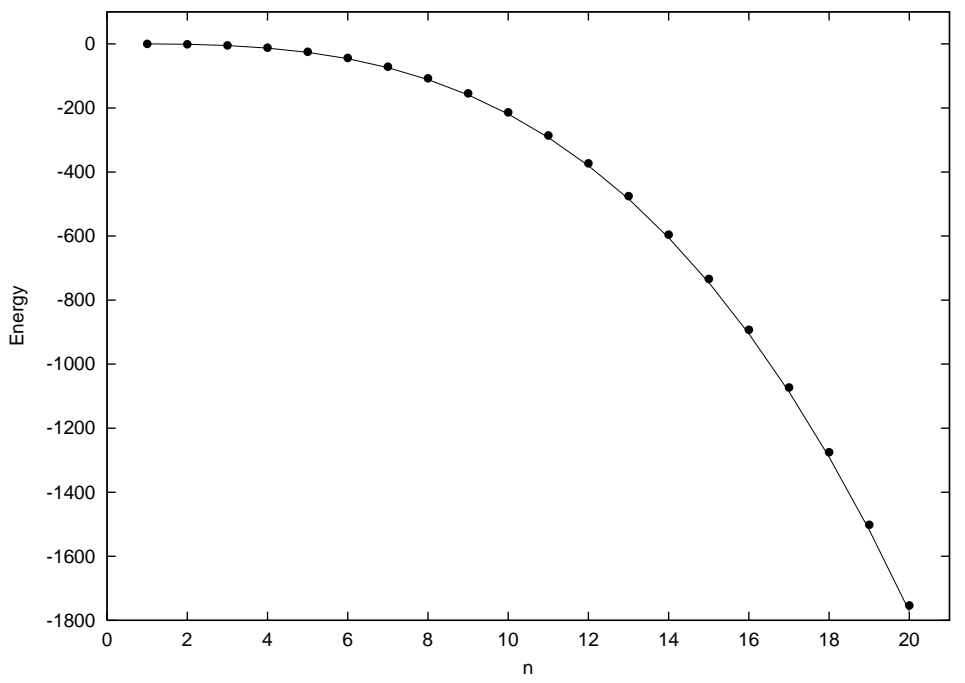

Figure 2: The energy as a function of $n$ (circles) and the estimate described in the text (curve).

proved about the extrema of this energy function and in particular there is the lower bound $[1]$

$$
U>\frac{1}{2}-\frac{2}{3} n^{2}
$$

Using this result in (2.14) we obtain a lower bound for $E_{\rho}$ which we can then minimize over the radius $\rho$, finding a minimum value at

$$
\rho=\frac{2 n}{3}-\frac{1}{2 n}
$$

to obtain the lower bound

$$
E_{\text {sphere }}>-\left(\frac{2}{9} n^{3}-\frac{1}{3} n+\frac{1}{8}\right)
$$

where $E_{\text {sphere }}$ denotes the energy $(2.13)$ (with $m_{k}=1$ ) under the restriction that all points lie at the same distance from the origin. Clearly when we drop this restriction we have no rigorous lower bound for the unrestricted energy (2.13) which is our main concern, but since our numerical results suggest that in all the minimal energy configurations the points lie very close to the surface of a sphere then we expect that the quantity in (2.18) will be a good estimate of the minimal energy value, though it will tend to be slightly lower. In fig. 2 we plot this estimate as the curved line. Clearly the above expectations appear to be realized, in that the estimate is close to the true value, but bounds it from below. In fig. 3 we compare the estimate (2.17) (curve) for the size of the configuration with the numerical values as measured by $r_{\max }$ (circles). Again it can be seen that the estimate is quite accurate.

The above discussion suggests that our minimal energy solutions are closely related to those which maximize the sum of the mutual separations for points on a sphere. For $n=2,3,4,8,12$ it is proved that the extremal configurations for this sphere problem are 


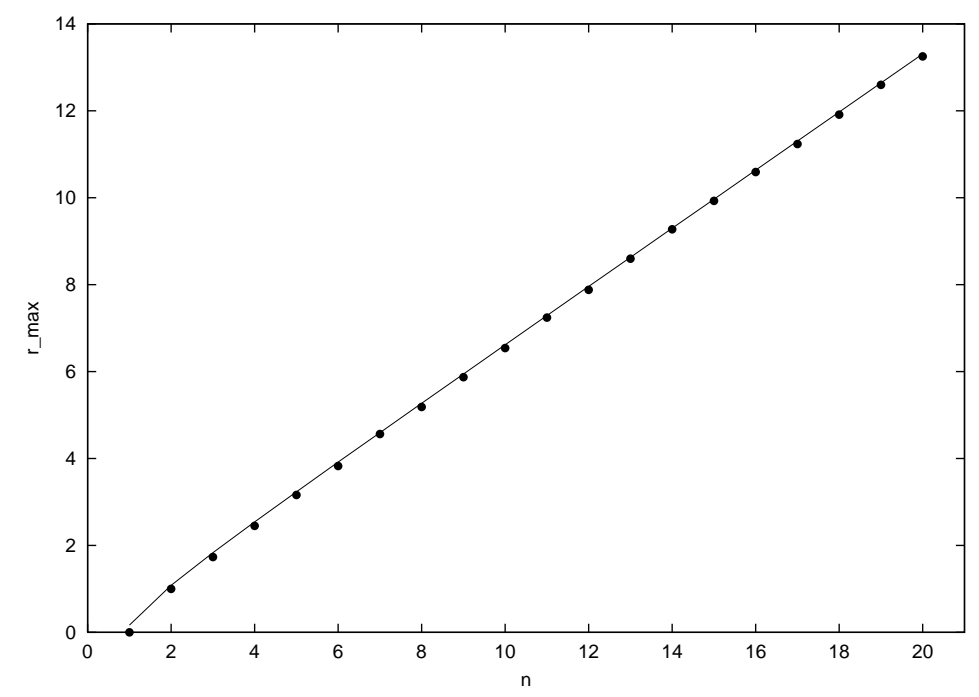

Figure 3: The size of the configuration $r_{\max }$ as a function of $n$ (circles) and the estimate $\rho$ described in the text (curve).

the dipole, triangle, tetrahedron, octahedron and icosahedron respectively (see [2] and references therein), which agrees with our results. For $n \leq 10$ this problem was investigated numerically by Berman and Hanes [7] and more recently a comprehensive numerical investigation has been performed by Rakhmanov, Saff and Zhou [22], who studied all $n \leq 200$ and obtained the symmetry groups of the extremal configurations. A comparison of the symmetry groups in Table 1 with those that appear in [22] reveals that the groups agree ${ }^{1}$ for all cases except $n=7$. In [22] the symmetry group for $n=7$ is given as $C_{2}$ and in [7] the configuration is described as two almost antipodal points with the remaining five points sprinkled around an equatorial band. Our configuration for $n=7$ is therefore consistent with a small deformation of the spherical extremal solution, which itself has little symmetry.

For all $1 \leq n \leq 20$ we found only one local minimum of the energy for each value of $n$, except for $n=16$. For $n=16$ the global minimum with $E=-892.7338$ has tetrahedral symmetry $T$, but we also found a local minimum with energy $E=-892.7256$ and symmetry $D_{4 h}$. Once again this mirrors the situation in studying extremal problems for points on a sphere [11].

In order to further investigate the similarites between our solutions and points on the sphere which maximize the sum of the mutual separations we turn our attention to configurations with icosahedral symmetry. For the sphere problem extremal configurations with icosahedral symmetry occur for a sequence of points [22] which begins $n=12,32,72, \ldots$ As we have seen, for $n=12$ our solution has icosahedral symmetry, with the points lying on the vertices of an icosahedron, so it is interesting to compute the minimal energy solutions for $n=32$ and $n=72$, to see if they are icosahedrally symmetric.

In fig. 4 we display the minimal energy configurations for $n=32$ and $n=72$, using the same method as in fig. 1. Both configurations contain a single shell and have icosahedral

\footnotetext{
${ }^{1}$ In [22] the symmetry group for 18 points should read $D_{4 d}$ not $D_{4 h}$.
} 

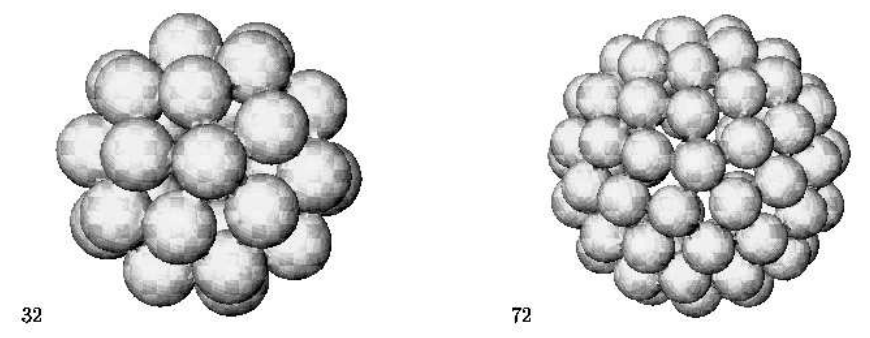

Figure 4: For $n=32$ and $n=72$ we display the icosahedrally symmetric minimal energy configurations by drawing spheres around the monopoles.

symmetry, as predicted by analogy with the sphere problem. For $n=32$ there are 12 points on the vertices of an icosahedron at a distance of 21.2516 from the origin and a further 20 points at a distance of 21.2680 from the origin. The associated polyhedron is the dual of a truncated icosahedron and the energy is $E=-7233.0539$. For $n=72$ the energy is $E=-82780.0335$. There are 12 points at a distance of 47.9338 and 60 points at a distance of 47.9563 .

\section{Distinct masses and $S U(2)$ monopoles}

In this section we mention two extensions of the study we have described so far. The first is the obvious generalization to monopoles which are not all of equal mass. As stated earlier, if all monopoles have the same mass then varying this mass produces the same configuration but scaled by the inverse common mass. Thus, the expectation for sets of distinct masses is that multiple shells will arise, with monopoles grouped into shells according to their mass, so that the heaviest monopoles sit closest to the origin, and with each set of monopoles in a given shell being arranged on the vertices of the polyhedron which arises in the minimization of the relevant number of equal mass monopoles. This nested polyhedron picture is consistent with the numerical results we have obtained. For example, in the case of two distinct masses and four monopoles of each mass, we set $m_{i}=1$, for $i=1, . ., 4$ and $m_{i}=2$, for $i=5, . ., 8$ the resulting configuration is that the four heavy monopoles sit on the vertices of a tetrahedron at a distance 1.5940 from the origin and the four light monopoles sit on the vertices of the dual tetrahedron scaled so that they are at a distance 6.3543 from the origin. As another example, with twelve light monopoles (with mass one) and six heavy monopoles (with mass two) the nested polyhedra are an octahedron and an icosahedron with scales 2.6954 and 13.8057 respectively, and oriented so as to preserve their common tetrahedral subgroups. Clearly the relative orientations of sets of nested polyhedra, together with their deformation as very distinct monopole masses are varied towards equality are interesting problems which are likely to need substantial 
investigation in each specific case.

The second extension we consider is to relate our results to the dynamics of well separated $S U(2)$ monopoles. Although, for $n>2$, the moduli space metric for $n S U(2)$ monopoles is not known explicitly, the asymptotic metric is known, which is valid in the region where all the monopoles are well separated [13]. This is the Gibbons-Manton metric and it is related to the Lee-Weinberg-Yi metric through some sign changes. Explicitly, the Gibbons-Manton metric is obtained by replacing equations (2.2)-(2.5) by the equations

$$
\begin{aligned}
g_{i i} & =m_{i}-\sum_{j \neq i} \frac{1}{\left|\mathbf{x}_{i}-\mathbf{x}_{j}\right|} \\
g_{i j} & =\frac{1}{\left|\mathbf{x}_{i}-\mathbf{x}_{j}\right|}, \quad i \neq j \\
\mathbf{W}_{i i} & =-\sum_{j \neq i} \mathbf{w}_{i j} \\
\mathbf{W}_{i j} & =\mathbf{w}_{i j}, \quad i \neq j
\end{aligned}
$$

If the approach of the previous section is now applied to this metric to find time-dependent homothetic solutions then, due to the sign changes, the upshot is that equation (2.10) is once again obtained, but with the replacement $C \mapsto-C$. In this case the physically acceptable solution is therefore to choose $C=-1$. With this choice both the function $\alpha(t)$ and the positions $\mathbf{y}_{k}$ that we have found to provide geodesics for the Lee-Weinberg-Yi metric carry over unchanged to produce geodesics of the Gibbons-Manton metric. The difference now is that these geodesics are only valid in the region where all the monopole are well separated, so the solutions break down before they can describe the collision of the monopoles.

For example, for $n=4$, the scaling geodesic describes the scattering of four monopoles on the vertices of a contracting tetrahedron. In fact, by using symmetry arguments, the full geodesic, to which this is a good approximation in the well separated regime, has been found and shows that as the monopoles approach they pass through a monopole solution with cubic symmetry and emerge on the vertices of an expanding tetrahedron which is dual to the incoming one [16]. For this example, even the metric is known exactly in terms of elliptic integrals [8].

It might be amusing to attempt to identify the outcomes of the various $S U(2)$ monopole scatterings that begin as the contracting polyhedra that we have identified, particularly those with high symmetry. However, a note of caution must be applied in this situation. The Gibbons-Manton metric possesses an $n$-torus isometry which the true monopole metric does not have for any finite separation. This means that the symmetry of a contracting polyhedron may only be realized in the true $S U(2)$ monopole solution at the limit of infinite separation. As an example of this situation consider the case $n=6$, where the contracting polyhedron is an octahedron. Using the one-to-one correspondence [18] between $S U(2)$ $n$-monopoles and (an equivalence class of) rational maps between Riemann spheres of degree $n$ we may determine the dimension of the moduli space of octahedrally symmetric $S U(2)$ monopoles of charge six. Degree six polynomials form the carrier space for the 7 - 
dimensional irreducible representation of $S U(2)$ and when this representation is restricted to the octahedral group it decomposes into irreducible representations of the octahedral group of dimensions one, three and three. Since two polynomials are required to form a rational map this shows that there are no octahedrally symmetric rational maps of degree six, and hence no octahedrally symmetric charge six monopoles. Thus six $S U(2)$ monopoles placed on the vertices of an octahedron break the octahedral symmetry for any finite value of the separation, no matter how large. This contrasts with the above mentioned case of $n=4$ with tetrahedral symmetry. The 5-dimensional irreducible representation of $S U(2)$ when restricted to the tetrahedral group decomposes into two 1-dimensional representations and a 3-dimensional representation. The basis polynomials for the two 1dimensional representations yield a 1-parameter family of tetrahedrally symmetric degree three rational maps, which corresponds to the geodesic describing the tetrahedral scattering of four monopoles [15]. Thus, in some cases the symmetry of the scaling geodesics of the Gibbons-Manton metric may be true symmetries of related geodesics in the true moduli space and in others they may not.

\section{Conclusion}

The scattering of $n$ distinct fundamental monopoles can be approximated by geodesic motion on the Lee-Weinberg-Yi manifold. We have described a variational method to construct some geodesics on this manifold for arbitrary values of $n$, and applied it to obtain a number of examples. The energy function used in this approach has features similar to that which arises in the classic problem of determining central configurations, but in contrast to central configurations it yields points which lie on a single shell for arbitrary values of $n$. The geodesics constructed by our method describe the scattering of monopoles on the vertices of a contracting, and then expanding, polyhedron, which generically is a deltahedron. We have found, and exploited, similarities between the deltahedra obtained here and those which arise in the problem of maximizing the sum of the mutual separations for points on a sphere.

\section{Acknowledgements}

We thank Ed Saff and Nikolay Andreev for useful correspondence and acknowledge advanced fellowships from PPARC (RAB) and EPSRC (PMS).

\section{References}

[1] R. Alexander, Acta Math. Acad. Sci. Hungar. 23, 443 (1972).

[2] N.N. Andreev, Proc. Stek. Inst. Math. 219, 20 (1997). 
[3] M.F. Atiyah and N.J. Hitchin, 'The geometry and dynamics of magnetic monopoles', Princeton University Press, 1988.

[4] M.F. Atiyah and P.M. Sutcliffe, Proc. R. Soc. A 458, 1089 (2002).

[5] R.A. Battye, G.W. Gibbons and P.M. Sutcliffe, 'Central Configurations in Three Dimensions', hep-th/0201101, to appear in Proc. Roy. Soc. (2002).

[6] M. Baus and J.-P. Hansen, Physics Reports 59, 1 (1980).

[7] J. Berman and K. Hanes, Math. Comp. 31, 1006 (1977).

[8] H. Braden and P.M. Sutcliffe, Phys. Lett. B 391, 366 (1997).

[9] S.A. Connell, 'The dynamics of $S U(3)$ charge $(1,1)$ magnetic monopoles', unpublished (1994).

[10] J.R. Edmundson, Acta Cryst. A48, 60 (1992).

[11] T. Erber and G.M. Hockney, Adv. Chem. Phys. 98, 495 (1997).

[12] L. Fejes Tóth, Acta Math. Acad. Sci. Hungar. 7, 397 (1956).

[13] G.W. Gibbons and N.S. Manton, Phys. Lett. B 356, 32 (1995).

[14] N.J. Hitchin, N.S. Manton and M.K. Murray, Nonlinearity 8, 661 (1995).

[15] C.J. Houghton, N.S. Manton and P.M. Sutcliffe, Nucl. Phys. B 510, 507 (1998).

[16] C.J. Houghton and P.M. Sutcliffe, Commun. Math. Phys. 180, 343 (1996).

[17] C.J. Houghton and P.M. Sutcliffe, Nucl. Phys. B 464, 59 (1996).

[18] S. Jarvis, J. Reine Angew. Math. 524, 17 (2000).

[19] K. Lee, E.J. Weinberg and P. Yi, Phys. Rev. D 54, 1633 (1996).

[20] J. Leech, Mathematical Gazette 41, 81 (1957).

[21] N.S. Manton, Phys. Lett. B 110, 54 (1982).

[22] E.A. Rakhmanov, E.B. Saff and Y.M. Zhou, 'Electrons on the sphere', in 'Computational Methods and Function Theory', World Scientific (1995).

[23] P. Rychenkova, 'Geometry of monopoles and domain walls', PhD Thesis, University of Cambridge (1999).

[24] D. Stuart, Commun. Math. Phys. 166, 149 (1994).

[25] P.M. Sutcliffe, Nucl. Phys. B 505, 517 (1997).

[26] E.J. Weinberg, Nucl. Phys. B 167, 500 (1980). 